\title{
Dimensão afetiva da pessoa com surdez adquirida, antes e após o implante coclear
}

\author{
Affective dimension of people with acquired deafness \\ before and after the use of cochlear implant
}

\author{
Midori Otake YAMADA \\ Maria Cecília BEVILACQUA²
}

\section{Resumo}

Este estudo teve como objetivo averiguar, antes e após o uso do implante coclear, a dimensão afetiva em pacientes adultos com surdez adquirida, no que diz respeito às modalidades dos sentimentos egoicos, sentimentos em relação ao próximo, sentimentos de temporalidade e estados de ânimo. Participaram 44 adultos que realizaram o implante coclear no Centro de Pesquisas Audiológicas do Hospital de Reabilitação de Anomalias Craniofaciais da Universidade de São Paulo de Bauru. Concluiu-se que, na vivência da surdez, houve predomínio de sentimentos negativos e de um clima afetivo de tensão e depressão, que levava o sujeito a uma vinculação negativa, de assintonia com o mundo. Entretanto, na vivência com o implante coclear, houve predomínio de sentimentos positivos e de um clima afetivo de tranquilidade e contentamento, observando-se uma vinculação positiva do sujeito e sintonia com o mundo.

Unitermos: Afeto. Implante coclear. Psicologia clínica. Surdez.

\begin{abstract}
The aim of this study was to investigate the affective dimension in adult patients with acquired deafness, both before and after using cochlear implants, by indicating the occurrence of changes in this dimension and investigating it, particularly in the modes of egoic feelings, feelings towards others, feelings of temporality and states of mind before and after the use of the cochlear implant. Forty-four adults took part in this study. They received cochlear implants from the Audiological Research Center at the Craniofacial Anomaly Rehabilitation Hospital at the University of São Paulo in Bauru. It was concluded that, during the period of deafness, negative feelings and an affective climate of tension and depression prevailed, leading the subject to a negative bonding, to a lack of syntony with the world. On the other hand, after the cochlear implant, positive feelings and an affective climate of tranquility and satisfaction prevailed, leading the subject to positive bonding, a feeling of syntony with the world.
\end{abstract}

Uniterms: Acquired deafness. Cochlear implant. Psychology. Affectivity.

A surdez adquirida traz um impacto avassalador na vida das pessoas, desestabilizando-as e alterando toda uma realidade construída até então, modificando suas relações e sua postura diante do mundo. Sem audição, a pessoa não tem informações dos eventos que estão fora do campo visual, fica à mercê do outro

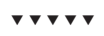

1 Universidade de São Paulo, Hospital de Reabilitação de Anomalias Craniofaciais, Centro de Pesquisas Audiológicas. R. Sílvio Marchione, 3-20, Vila Universitária, 17012-900, Bauru, SP, Brasil. Correspondência para/Correspondence to: M.O. YAMADA. E-mail: <miotake@centrinho.usp.br>.

2 Universidade de São Paulo, Faculdade de Odontologia de Bauru. Bauru, SP, Brasil. 
para realizar a leitura orofacial; a comunicação torna-se difícil, o que propicia o isolamento e favorece a depressão (Kerr \& Cowie, 1997; McKenna, 1995).

Revisando estudos sobre a interação da pessoa com surdez e o ouvinte, Rutman e Boisseu (1995) observaram que a surdez afeta negativamente as relações interpessoais. As dificuldades na comunicação desencadeiam constrangimento, frustração e incômodo, tanto na pessoa com surdez como no ouvinte.

As consequências da surdez adquirida na vida adulta são amplas e não podem ser entendidas somente pela perda auditiva detectada nos exames audiológicos. O sujeito vivencia a perda: o desamparo e as restrições na área pessoal e social afetam sua qualidade de vida (L.R. Halberg, U. Halberg \& Kramer, 2008; Helvik, Jacobsen \&Halberg, 2006; Yamada \& Bevilacqua, 2005)

O implante coclear tem sido indicado como recurso benéfico e efetivo para pessoas com deficiência auditiva severa/profunda que não tenham benefícios com o aparelho de amplificação sonora individual. Representa uma nova tecnologia para pessoas com deficiência auditiva incapazes de compreender a fala.

O implante coclear é uma prótese auditiva, composta de componentes internos e externos, que substitui o órgão sensorial da audição. O componente interno é implantado mediante intervenção cirúrgica na qual os eletrodos são inseridos na cóclea (ouvido interno). O componente externo, por meio de um microfone instalado junto à orelha, capta o som, que é transmitido por um fio ao processador de fala. O processador envia a informação codificada para uma antena transmissora, colocada junto ao receptor-estimulador, excitando o nervo auditivo (Costa Filho \& Bevilacqua, 2006; Costa Filho, Bevilacqua, Amantini \& Lamônica Neto, 2002).

De maneira ampla, a perda da audição ocasiona um abalo profundo e traz consequências negativas à vida daqueles que passam por esse processo, alterando-Ihes o modo de ser e de existir no mundo.

Com o implante coclear, a possibilidade de ouvir é restaurada. Surgem, então, algumas questões importantes a respeito da pessoa que, um dia, tendo sido ouvinte, passou a ser surda e, posteriormente, passou a ouvir novamente, por meio de um implante coclear. 64 Pode-se perguntar: como é ser uma pessoa com um implante coclear? como fica a afetividade? quais os sentimentos predominantes antes e após o uso do implante? como se configura o estado de ânimo nesses dois momentos?

Alguns estudos indicam influências significativas após o implante coclear, nas condições de vida diária, graças à melhora na comunicação que gera um incremento na qualidade de vida (Maillet, Tyler \& Jordan, 1995; Mortensen, Jochumsen, Pedersen \& Ovesen, 2004; Zhao, Stephens, Sim \& Meredith, 1997). Os benefícios psicossociais, como o aumento de felicidade, da autoconfiança e uma maior independência, assim como a diminuição dos sentimentos de ansiedade, angústia e isolamento, foram relatados nos estudos de Hogan (1997) e Kou, Shipp e Nedzelski (1994).

Ao investigar o grau de satisfação do paciente e o implante coclear, Ito, Takagi, Kawano e Honjo (1995) e Murakami, Neme, Yamada e Bevilacqua (2001) demostraram que, embora alguns pacientes manifestassem algum tipo de incômodo quanto à estética, o implante os ajudava a ouvir, a melhorar a qualidade de vida e a ficarem mais satisfeitos.

No entanto, poucas são as publicações na área da Psicologia com relação a esse assunto, e não foram encontradas pesquisas sobre afetividade da pessoa com surdez adquirida ou usuária do implante coclear que apresentassem proposta semelhante à deste estudo.

Considerando a afetividade ou a dimensão afetiva como uma das dimensões básicas da existência humana, e em razão de o próprio fenômeno destacar-se nos relatos dos pacientes atendidos no Centro de Pesquisas Audiológicas do Hospital de Reabilitação de Anomalias Craniofaciais da Universidade de São Paulo, campus de Bauru (HRAC-USP-Bauru), optou-se pela investigação do tema com base na concepção existencial, fundamentada em Emílio Romero.

Alguns teóricos foram consultados sobre o tema da afetividade: Laplanche e Pontalis (1983), psicanalistas: Arnold, Eysenck e Meili (1994), comportamentalistas: e Doron e Parot (1998), ecléticos. Esses autores apresentaram os conceitos fundamentais, porém a distinção entre emoção, sentimento, estados de ânimo ou humor e paixão não ficou clara.

Romero (1998) oferece uma sistematização e caracterização sustentável dos conceitos, com defi- 
nições claras a respeito do tema. A afetividade ou dimensão afetiva, segundo Romero (1998; 2002), é a capacidade de o homem afetar-se subjetivamente com o que acontece. Os eventos têm uma ressonância e um significado próprio, que indicam o modo como a pessoa vincula-se a si mesma e ao mundo.

O autor postula que a afetividade permeia todas as dimensões da existência e está presente na vida do ser humano de um modo envolvente e intenso, como nas emoções; de maneira sutil, em certos estados de ânimo; e ainda em forma de vínculos negativos ou positivos, nos sentimentos. Ou seja, o tipo de relação mantida com os objetos e as pessoas pode ter um caráter positivo, quando aproxima e constrói, e um caráter negativo, quando distancia e destrói. Sendo assim, o sentimento é uma das maneiras de o homem experienciar suas relações - positiva ou negativamente - e de atribuir significações ao vivido, que passam a constituir o seu modo de ser (Romero, 1998; 1999; 2002).

Os estados de ânimo - ou de humor, como também são chamados - constituem o clima vivencial dominante, assim como existe um clima físico, com sua temperatura e luminosidade, igualmente existe um clima subjetivo. São os estados de ânimo que estabelecem uma disposição geral que leva a pessoa a enxergar o mundo de uma determinada maneira, a ter um modo predominante de ver a realidade. Romero (1998; 1999; 2002) propôs uma classificação dos estados de ânimo em dois grandes eixos, que se dividem em duas tendências contrapostas, embasados no centro vivencial da pessoa. Eles situam-se no eixo do abatimento versus exaltação e no eixo da tranquilidade ou relaxamento versus tensão.

Neste estudo, embasado na concepção de Emílio Romero, deu-se enfoque aos sentimentos e aos estados de ânimo da pessoa com surdez adquirida e usuária do implante coclear, por caracterizarem o mundo e o modo como elas se encontram.

Diante da frequência desse fenômeno nos relatos dos pacientes que confirmam a importância da afetividade na vida das pessoas, este estudo teve como objetivos averiguar, antes e após o uso do implante coclear, a dimensão afetiva em pacientes com surdez adquirida, e investigar as modalidades dos sentimentos egoicos, sentimentos em relação ao próximo, sentimentos de temporalidade e estados de ânimo ou humor.

\section{Método}

\section{Participantes}

Os participantes do estudo foram 44 adultos de ambos os sexos, na faixa etária entre 21 e 68 anos, com ensino fundamental completo, com deficiência auditiva pós-lingual, usuários do implante coclear e pacientes do programa de implante coclear do Centro de Pesquisas Audiológicas do HRAC-USP-Bauru. O estudo foi aprovado pelo Comitê de Ética em Pesquisa em Seres Humanos do HRAC/USP, protocolo no 124/98, em 30/09/1998.

\section{Instrumento}

Foi utilizado um questionário como instrumento de investigação, elaborado e sistematizado segundo o referencial teórico adotado (Kou et al., 1994; Romero, 1998) e o modelo do Questionário de Autoavaliação, Inventário de Ansiedade Traço-Estado (IDATE), de Spielberg, Gorsuch e Lushene (1979), que passou por estudo piloto e alterações.

\section{Procedimentos}

As afirmações do questionário foram desenvolvidas com base nos relatos mais comuns de pacientes com surdez adquirida e também nos relatos dos que realizaram o implante coclear e que foram atendidos durante entrevistas psicológicas realizadas pela pesquisadora no período de nove anos no atendimento do Programa de Implante Coclear do Centro de Pesquisas Audiológicas do HRAC-USP-Bauru. As afirmações visaram a verificar a dimensão afetiva no que se refere aos sentimentos e estados de ânimo. Para alcançar os objetivos propostos, o questionário dividiu-se em duas partes (Parte I e Parte II), com 21 afirmações cada uma. A Parte I referia-se à experiência de vida antes do implante coclear, e a Parte II, após o implante coclear. Cada afirmação do questionário tinha correspondência com uma escala de 0-5 pontos ("nunca","raramente", "às vezes", "frequentemente" $e$ "sempre"). Definiu-se que uma maior pontuação corresponderia à piora na dimensão afetiva do sujeito, isto é, na sua vinculação com o mundo, de modo mais negativo. Para a análise estatística dos resultados da dimensão afetiva pré- e pós-uso do implante coclear, foi utilizada a prova não-paramétrica de Wilcoxon Siegel (Siegel, 1977). 


\section{Resultados e Discussão}

Quanto aos sentimentos egoicos (egodistônico e egossintônico), os resultados mostraram que houve uma diferença estatisticamente significativa entre o pré e pós-uso do implante coclear. Os resultados obtidos para os sentimentos ego-distônicosforam: média $=\mu \mathrm{T}=495$; desvio-padrão $=\sigma \top=85,69 ;$ valor padronizado $=Z e=-5,76$. $\mathrm{O}$ valor padronizado $(\mathrm{Ze}=-5,76)$ levou à rejeição da $\mathrm{HO}$ em favor da $\mathrm{H} 1$, sendo $\mathrm{HO}$ a hipótese de não alteração dos sentimentos ego-distônicos e $\mathrm{H} 1$ a hipótese alternativa.

Os resultados obtidos para os sentimentos egossintônicos foram: média $=\mu \mathrm{T}=495$; desvio-padrão $=\sigma \top=85,69$; valor padronizado $=Z e=-5,78$. $O$ valor padronizado $(\mathrm{Ze}=-5,78)$ também levou à rejeição da $\mathrm{HO}$ em favor da $\mathrm{H} 1$; sendo $\mathrm{HO}$ a hipótese de não alteração dos sentimentos egossintônicos e H1 a hipótese alternativa.

A investigação quanto aos sentimentos egoicos, postulada por Romero (1998; 2002) como sentimentos em relação à própria pessoa, ao seu mundo pessoal e íntimo, mostrou melhora estatisticamente significante após o uso do implante coclear. Antes do uso do implante coclear, os sentimentos egoicos estavam direcionados para sentimentos egodistônicos, que se orientavam para a autodesvalorização e sofrimento, tais como: inferioridade, solidão, tristeza, angústia, depressão, desânimo, insatisfação, desvalor de si mesmo, rejeição, vazio e isolamento. Os resultados obtidos foram semelhantes aos da literatura especializada consultada, que indicaram a predominância desses sentimentos em pessoas que perderam a audição (Kerr \& Cowie, 1997; McKenna, 1995; Rutman \& Boissseu, 1995; Yamada \& Bevilacqua, 2005). A predominância de sentimentos negativos leva o sujeito a uma vinculação negativa com as pessoas e com a vida de modo geral e faz com que atribuam significações negativas ao vivido (Romero, 1998; 2000; 2002). Isso implica uma diminuição, um abalo, tanto para o ego do sujeito quanto para sua disponibilidade existencial. Quando esses sentimentos são vivenciados de maneira acentuada ou prolongada, podem levar a formas de ser malogradas, a um profundo desencontro consigo mesmo.

A experiência com a surdez passa a ser fonte de 66 sofrimento, destruindo os referenciais que a pessoa tinha até então e que serviam de orientação para sua vida. Constata-se, assim, que o impacto da deficiência auditiva não pode ser entendido simplesmente pela medida da sua intensidade e limitações objetivas que a deficiência impõe (Halberg et al., 2008; Helvik et al., 2006; Kerr \& Cowie, 1997). Ela muda todo o rumo da vida da pessoa e todo o seu ser no mundo é alterado.

Por outro lado, as experiências positivas afirmam e ratificam a autoconfiança, o que foi possível comprovar nos sujeitos usuários do implante coclear deste estudo, pois os resultados indicaram que os sentimentos egoicos estavam direcionados para os sentimentos egossintônicos, que se orientam para a satisfação do eu. Neste momento, os sentimentos foram de: felicidade, sentimento do próprio valor, autoconfiança, autoaceitação, segurança, contentamento, alegria, cordialidade, satisfação e disposição.

Na literatura consultada, existem pesquisas que confirmam os resultados encontrados neste estudo (Hogan, 1997; Knutson et al., 1998; Kou et al., 1994; Maillet et al., 1995; Mortensen et al., 2004; Murakami et al., 2001; Tyler, 1994; Zhao et al., 1997).

Esses sentimentos positivos, que confirmam a potencialidade e o reconhecimento de si mesmo como agente de seu valor, indicam que a pessoa está satisfeita com sua capacidade, seu modo de agir e que apresenta um razoável grau de autoestima (Romero, 1994). Desta maneira, verificou-se que ouvir através do implante coclear devolve a confiança perdida do sujeito, que passa a acreditar novamente em si, sentindo-se mais seguro. Com um bom grau de autoconfiança, a pessoa apresenta capacidade para enfrentar dificuldades, realizar tarefas e superar limitações e barreiras.

Quanto aos sentimentos em relação ao próximo, os resultados mostraram que houve uma diferença estatisticamente significativa entre o pré e pós-uso do implante coclear. Os resultados obtidos foram: média $=\mu \top=495$; desvio-padrão= $\sigma \mathrm{T}=85,69$; valor padronizado $=Z e=-5,78$. O valor padronizado $(Z e=-5,78)$ levou à rejeição da $\mathrm{HO}$ em favor da $\mathrm{H} 1$; sendo $\mathrm{HO}$ a hipótese de não alteração dos sentimentos em relação ao próximo e H1 a hipótese alternativa.

Esses resultados indicaram alterações positivas após o uso do implante coclear, pois os sujeitos passaram a vincular-se positivamente, entabulando movimentos 
de aproximação do outro. Se, na vivência da surdez, o vínculo com o outro tinha um caráter negativo, de distanciamento, mágoa e desconfiança (McKenna, 1995; Rutman \& Boisseu, 1995), com o uso do implante, o vínculo em relação ao outro foi de caráter positivo e de aproximação, e os sentimentos mais frequentes foram de simpatia e sintonia (Hogan, 1997; Maillet et al., 1995; Tyler, 1994; Zhao et al., 1997).

Esse movimento existencial de aproximação e sintonia com o outro abre um espaço comum e compartilhável da pessoa usuária do implante coclear com os ouvintes em geral, o que muda o valor do contato interpessoal, que passa a ser gratificante.

Melhorando a comunicação no cotidiano, o sujeito pode participar mais das atividades sociais, ampliando suas possibilidades, aproximando-se do outro, sentindo-se integrado a uma comunidade, o que o faz sentir que não é um indivíduo isolado.

Com relação aos sentimentos de temporalidade, os resultados também mostraram uma diferença estatisticamente significativa entre o pré e pós-uso do implante coclear. Os resultados obtidos foram: média $=\mu T=495$; desvio-padrão= $\sigma \top=85,69$; Valor Padronizado=Ze=-5,24. O valor padronizado $(Z e=-5,24)$ levou à rejeição da $\mathrm{HO}$ em favor da $\mathrm{H} 1$, sendo $\mathrm{HO}$ a hipótese de não alteração dos sentimentos de temporalidade e H1 a hipótese alternativa.

No que diz respeito aos sentimentos de temporalidade - o modo de sentir o tempo, a experiência do sujeito em vivenciá-lo na sua relação com o mundo (Romero, 1998; 2002) - os resultados também indicaram alterações positivas após o uso do implante, com diferenças estatisticamente significantes. Antes do implante, os sujeitos apresentavam maior preocupação com o futuro e o sentimento de espera era angustiante. Naquele momento, o futuro já não era mais um lugar de realizações, tornava-se desconhecido e sem perspectivas. 0 futuro apareceu como fracasso dos valores a que aspiravam. No geral, após o implante coclear, observou-se uma diminuição da preocupação com o futuro: este passou a ter mais possibilidades, tornando-se um campo possível de realizações.

Quanto aos estados de ânimo, os resultados indicaram que houve uma diferença estatisticamente significativa entre o pré e pós-uso do implante coclear.
Os resultados obtidos foram: média $=\mu T=495$; desvio- padrão= $\sigma \top=85,69$; valor padronizado=Ze=-5,78. 0 valor padronizado ( $\mathrm{Ze}=-5,78)$ levou à rejeição da $\mathrm{H0}$ em favor da H1, sendo $\mathrm{HO}$ a hipótese de não alteração dos sentimentos em relação ao próximo e H1 a hipótese alternativa.

Os resultados, no que se refere aos estados de ânimo, evidenciaram também melhoras estatisticamente significantes após o uso do implante. Antes, o clima vivencial dominante dos sujeitos era de tensão e depressão (McKenna, 1995; Romero, 1991; 1994); após o uso do implante, constatou-se uma transformação em relação aos estados de ânimo, indicando alterações positivas: o clima vivencial dominante passou a ser de mais tranquilidade, serenidade e contentamento (Harris, Anderson \& Novak, 1995; Ito et al., 1995; Kou et al., 1994; Mortensen et al., 2004; Murakami et al., 2001; Tyler, 1994; Zhao et al., 1997).

Os resultados gerais obtidos neste estudo, que revelaram uma diferença estatisticamente significante antes e após o uso do implante coclear (média $=\mu \top=495$; desvio-padrão= $\sigma T=85,69$; valor padronizado=Ze=-5,78), indicam que os sujeitos apresentaram alterações positivas quanto à dimensão afetiva após o uso do implante; ou seja, melhoras quanto aos sentimentos e estados de ânimo (Quadros 1 e 2).

É importante ressaltar, aqui, a influência da dimensão afetiva nas outras sete dimensões da existência (dimensão social e interpessoal, corporal, da práxis, motivacional, valorativa, temporal e espacial). Partindo-se do pressuposto de que todas elas se entrecruzam, influenciando-se mutuamente e com predominância variável entre si (Romero, 1998; 2002), é possível afirmar que podem trazer modificações gerais na pessoa como um todo. Nesse sentido, pela extensão e complexidade de todas as outras dimensões do estudo, sugere-se que sejam realizadas investigações posteriores a respeito das demais dimensões do existir humano.

Acredita-se que as alterações encontradas neste estudo - na dimensão afetiva dos adultos com surdez adquirida que realizaram o implante coclear - devem-se aos benefícios proporcionados pelo implante. No entanto, não se pode negar a participação do próprio paciente, suas expectativas e possibilidades físicas, emocionais e sociais; o trabalho em equipe interdisciplinar; a visão do paciente na sua totalidade; e, 
Quadro 1. Sentimentos identificados antes e após o implante.

\begin{tabular}{lll}
\hline Sentimentos & Pré-implante & Pós-implante \\
\hline Egoicos & Inferioridade, solidão,desânimo, tristeza, desvalor, & Felicidade, próprio valor, autoconfiança, segurança, \\
& depressão, rejeição, angústia, insatisfação, vazio & cordialidade, satisfação, disposição \\
Em relação ao próximo & Vínculo negativo & Vínculo positivo \\
& Movimento de distanciamento & Movimento de aproximação \\
& Mágoa, desconfiança & Simpatia, sintonia \\
De temporalidade & Maior preocupação com o futuro & Menor preocupação com o futuro \\
& Espera/angústia & Sentimentos positivos/tranquilos \\
& Vivência do tempo angustiante, ameaçadora e longa & Vivência do tempo otimista/curta \\
\hline
\end{tabular}

Quadro 2. Estados de ânimo identificados antes e após o implante.

\begin{tabular}{lll}
\hline Estados de ânimo & Pré-implante & Pós-implante \\
\hline Implante coclear vivencial & Tensão/depressão & Tranquilidade/contentamento \\
Disposição geral & Não sintonia/mundo & Bem-estar e sintonia/mundo \\
\hline
\end{tabular}

fundamentalmente, a filosofia que permeia o hospital "Centrinho", focada no ser humano, que pode ter uma influência no significado positivo que os pacientes atribuem ao processo de ouvir.

\section{Considerações Finais}

Este estudo permitiu concluir que ocorreram mudanças na afetividade do adulto com surdez adquirida após o uso do implante coclear. A surdez e o implante coclear provocaram mudanças na vinculação do sujeito com o mundo, exercendo influência nos climas afetivos que orientavam sua existência. Enquanto a vivência da surdez estimulou o predomínio de sentimentos negativos com relação a si mesmo e ao outro, com predomínio de uma vinculação negativa, de assintonia com o mundo e de um clima afetivo de tensão e depressão, o uso do implante coclear e a possibilidade de reviver no mundo sonoro proporcionaram o predomínio de sentimentos positivos em relação a si mesmo e ao outro, evidenciando uma vinculação positiva, de sintonia com o mundo e de um clima afetivo de tranquilidade e contentamento.

Deste modo, para o paciente, ouvir por meio do implante coclear significou estar mais integrado consigo mesmo e poder se relacionar de uma maneira mais completa com as pessoas e com o mundo.
O referencial teórico propiciou a exploração e a compreensão dos fenômenos afetivos tratados neste estudo, confirmando a importância dos afetos e da afetividade como uma dimensão básica da existência.

\section{Referências}

Arnold W., Eysenk H. J., \& Meili R. (1994). Dicionário de psicologia. São Paulo: Loyola.

Costa Filho, O. A., \& Bevilacqua, M. C. (2006). Implantes cocleares. In S. S. Costa. Otorrinolaringologia: princípios e práticas (2a ed., pp.447-453). Porto Alegre: Artmed.

Costa Filho, O. A., Bevilacqua, M. C., Amantini, R. C. B., \& Lamônica Neto, D. (2002). Implante coclear em adultos. In C. A. H. Campos \& H. O. O. Costa. Tratado de otorrinolaringologia (pp.278-289). São Paulo: Roca.

Doron, R., \& Parot, F. (1998). Dicionário de psicologia. São Paulo: Ática.

Hallberg, L. R., Hallberg, U., \& Kramer, S. E. (2008). Self-reported hearing difficulties, communication strategies and psychological general well-being (quality of life) in patients with acquired hearing impairment. Disability and Rehability, 30 (3), 203-212.

Harris, J. P., Anderson, J. P., \& Novak, R. (1995). An outcomes study of cochlear implants in deaf patients: audiologic, economic and quality-of-life changes. Archives of Otolaryngology: Head \& Neck Surgery, 121 (4), 398-404.

Helvik, A. S., Jacobsen, G., \&Halberg, L. R. (2006). Psychological well-being of adults with acquired hearing impairment. Disability and Rehability, 28 (9), 535-545. 
Hogan, A. (1997). Implant outcomes: towards a mixed methodology for evaluating the efficacy of adult cochlear implant programmes. Disabilility and Rehability, 19 (6), 235-243.

Ito, J., Takagi, A., Kawano, M., \& Honjo, I. (1995). Studies of currently used and experimental cochlear implants. Acta Oto-Laryngologica, 115 (4), 493-496.

Kerr, P. C., \& Cowie, R. I. D. (1997). Acquired deafness: a multi-dimensional experience. British Journal of Audiology, 31 (3), 177-188

Knutson, J. F., Murray, K. T., Husarek, S., Westerhouse, K., Woodworth, G., Gantz, B. J., et al. (1998). Psychological change over 54 months of cochlear implant use. Ear and Hearing, 19 (3), 191-201.

Kou, B. S., Shipp, D. B., \& Nedzelski, J. M. (1994). Subjective benefits reported by adult nucleus 22-channel cochlear implant users. The Journal of Otolaryngology, 23 (1), 8-14.

Laplanche J., \& Pontalis J. B. (1983). Vocabulário da psicanálise (7a ed.). São Paulo: Martins Fontes.

Maillet, C. J., Tyler, R. S., \& Jordan, H. N. (1995). Change in the quality of life of adult cochlear implant patients. The Annals of Otology, Rhinology \& Laryngology Supplement, $165,31-48$.

McKenna, L. (1995). Alguns aspectos psicológicos da surdez. In J. Ballantyne, M. C. Martin \& A. Martin. Surdez (5a ed., pp.227-232). Porto Alegre: Artes Médicas.

Mortensen, M. V., Jochumsen, U., Pedersen, C. B., \& Ovesen, T. (2004). Cochlear implants to deaf adults: psychosocial consequences. Ugeskrift for Laeger, 166 (34), 2894-2898.

Murakami, G. A. O., Neme, C. M. B., Yamada, M. O., \& Bevilacqua, M. C. (2001). Expectativas prévias ao implante coclear e avaliação pós implante em adolescentes. Estudos de Psicologia (Campinas), 18 (2), 5-16.

Romero, E. (1991). Estações e climas afetivos: os estudos de ânimo. São Paulo: SOBRAPHE.

Romero, E. (1994). Oinquilino do imaginário: formas de alienação e psicopatologia. São Paulo: Lemos.

Romero, E. (1998). As dimensões da vida humana: existência e experiência. São José dos Campos: Novos Horizontes.
Romero, E. (1999). Neogênese: o desenvolvimento pessoal mediante a psicoterapia. São José dos Campos: Novos Horizontes.

Romero, E. (2000). As dimensões da existência como fundamento para um abordagem compreensiva e fenomenológica em psicologia. In D. S. P. Castro, F. P. Ázar, J. D. Piccino \& R. S. Josgrilberg. Fenomenologia e análise do existir (pp.165-178). São Paulo: Universidade Metodista de São Paulo.

Romero, E. (2002). As formas da sensibilidade: emoções e sentimentos na vida humana ( $2^{\mathrm{a}}$ ed.). São José dos Campos: Della Bídia.

Rutman, D., \& Boisseau, B. (1995). Acquired hearing loss: social and psychological issues and adjustment processes. International Journal of Rehabilitation Research, 18 (4), 313-323.

Siegel, S. (1977). Estatística não paramétrica para as ciências do comportamento. São Paulo: McGraw-Hill do Brasil.

Spielberger, C. D., Gorsuch, R. L., \& Lushene, R. E. (1979). Inventário de ansiedade traço-estado-idade. Rio de Janeiro: Cepa.

Tyler, R. S. (1994). Advantages of disadvantages expected and reported by cochlear implant patients. The American Journal of Otology, 15 (4), 523-531.

Yamada, M. O., \& Bevilacqua, M. C. (2005). O papel do psicólogo no programa de implante coclear do Hospital de Reabilitação de Anomalias Craniofaciais. Estudos de Psicologia (Campinas), 22 (3), 255-262. doi: 10.1590/S010 3-166X2005000300004

Zhao, F., Stephens, S. D., Sim, S. W., \& Meredith, R. (1997). The use of qualitative questionnaires in patients having and being considered for cochlear implants. Clinical Otolaryngology and Allied Sciences, 22 (3), 254-259.

Recebido em: 31/8/2009

Versão final reapresentada em: 4/7/2011

Aprovado em: 23/8/2011 
